Volume 9, No.3, May - June 2020

International Journal of Advanced Trends in Computer Science and Engineering

Available Online at http://www.warse.org/IJATCSE/static/pdf/file/ijatcse207932020.pdf

https://doi.org/10.30534/ijatcse/2020/207932020

\title{
Classification of Coronary Heart Artery Disease Using IVUS Images by SVM Classifier with Modified Radial Basis Function Kernel (MRBFK)
}

\author{
S. Sridevi ${ }^{1}$, M. Sundaresan ${ }^{2}$ \\ ${ }^{1}$ Research Scholar, Department of Information Technology, Bharathiar University, Coimbatore, India \\ devisris.mphil@gmail.com \\ ${ }^{2}$ Professor and Head, Department of Information Technology, Bharathiar University, Coimbatore, India \\ bu.sundaresan@gmail.com
}

\begin{abstract}
In real world phenomenon, Digital image processing techniques and algorithms are widely used in the medical applications. The medical applications are used to predict the disease for reducing the death rate. But, nowadays heart disease is one of the most significant and exigent problem. Based on the problem, heart disease can be in different form like atherosclerotic plaque, calcium accumulation, and vice versa. Here this paper concentrates only on atherosclerotic plaque, which is the major cause of coronary heart artery diseases and human deaths. Coronary heart artery plaque layer analysis is one of the most important and challenging problems in real world phenomenon. For this purpose, this paper elucidates and presents a five phased approach of (1) Cartesian to Polar (C2P) Coordinate conversion (2) Iterative level Energy Computation Method (IECM) (3) Feature Extraction (4) Classification and (5) Performance Evaluation. Hence, the performance is analyzed for classification of IVUS images based on Sensitivity, Specificity and Precision. The proposed method achieves Accuracy of $88 \%$ and the Kappa value of 0.92 which indicates the good agreement test using SVM Classifier with Modified Radial Basis Function Kernel (MRBFK).
\end{abstract}

Key words: Atherosclerosis, Coronary Heart Artery, Classification, Feature Extraction, Lumen diameter, Shape Parameter.

\section{INTRODUCTION}

Health care systems are based on the progressive information technology to electronically accumulate process, scrutinize, allocate, demonstrate and store the patient data [1]. The increasing volume of medical data demands new techniques for organizing, sharing, managing and extracting knowledge.

The knowledge extracted can be further used for diagnosing disease, preventing human error and informing about the functions and consequences of several diseases. Moreover, advances in digital image processing system and more specifically, image analysis takes a major hand in medical progress, as well as analysis of medical images play an ever-increasing crucial role in terms of research and medical diagnosis [2]-[4].

Coronary Angiography is a standard method for medical imaging procedures and diagnosis of coronary heart disease [5]. Angiogram technology does not provide the heart artery layer and vessel wall information for diagnostic procedure. It is mainly failed to assess the plaque layer boundary, which is responsible for partial or total obstruction of the arteries [6]. Nowadays, Intravascular Ultrasound (IVUS) has been introduced as an alternative technique to angiography diagnostic aiming at more accurate imaging of coronary atherosclerosis. The specific feature extraction is necessary in IVUS images for accurate morph metric analysis of coronary plaques and accordingly the assessment of the atherosclerotic lesion length [7]-[9].

Cardiac disease research is the most important areas in the field of medicine and also that contains the advantages of several existing technologies [10]. Predominantly coronary heart artery disease and atherosclerosis are worldwide fatal diseases for both men and women among all ethnic groups. American Heart Association says the ratio of death rate is mainly increased based on cardiac diseases. Atherosclerosis involves a build-up on the inner side of artery walls [11]. Heart arteries are supplies the blood to the heart. Normal artery contains the 
normal blood flow through the artery and up normal arteries contains the up normal blood flow through the artery which is usually leading to a heart attack and cardiac arrest to the blood supply. There is overwhelming evidence that high blood cholesterol increases the risk of developing atherosclerosis [12]-[15].

This system is also able to perform risk classification of patients based on the semantically structured information of patient data. And also contains the detection of luminal and medial-adventitial boundaries, based on the results of neighbor pixel analysis, performed by means of a Kernalized function, and on a smoothing step using Radial Basis Function (RBF) approximation [16].

\section{LITERATURE SURVEY}

Ji Yang, Mehdi Faraji [17] described the FCN based deep architecture for automatic segmentation of IVUS frames and also reconstructing the human arteries. Deep architecture using a smaller number of training images and achieves the higher degree of generalization ability and also strengthen the augmentation process. Training images are evaluated with commonly used parameters like Jaccard measure and Hausdorff distance. The above said method not evaluated the other standard parameter like specificity, precision accuracy.

Lucas Lo Vercio, Jose Ignacio Orlando, Mariana del Fresno, and Ignacio Larrabide [18] presented a automatic segmentation of luminal boundary by means different image features. Image filters, edge detectors and textual descriptors were taken for the assessment. The assessment is based on classification techniques. SVM classifier used to training the feature set and AUC and Jaccard index measurement.

VaishaliNaik, R.S.Gamad and P.P Bansod [19] explained the segmentation of the common carotid artery and determination of the Intima-media thickness based on the commonly used methods are dynamic programming techniques, Hough transform, Nakagami mixture modeling, active contour and edge detection and tracking method. And then performance is validated by different parameter metrics.

Huang Yi; Yan, Wenjun; Xia, and Menghua [20] explained a special mask based on the geometric property of media and an assumption about the intensity distribution. For media segmentation, the guided multi scale normalized cut extract the Region of Interest (ROI). Then a target binarization processing can extract the rough lumen region of ROI. Geometrical and binarization methods are used in the ROI extraction. Based on the observation the above system concentrates only on Masking and segmentation process. The above limitation can be overcome by using the following proposed method.

The paper is organized as follows: Literature survey is described in section 2. Proposed methodology are detailed in section 3 followed by the statistical analysis and performance evaluation. In section 4 described the results and discussion and comparison results are in section 4.1. Finally, the conclusion and future enhancement are given in section 5 .

\section{MATERIALS AND METHODS}

The flow chart of the proposed method for classification of coronary heart artery disease using IVUS image is shown in Figure 1.

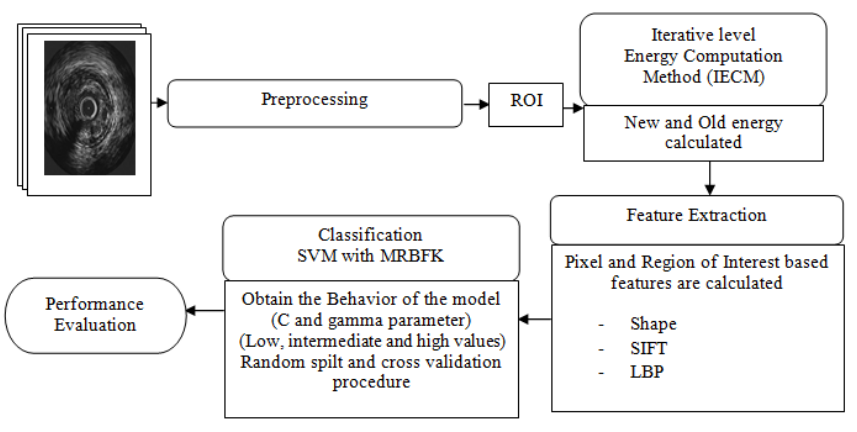

Figure 1: Flow chart of proposed method

\subsection{Image Acquisition}

Image acquisition is the first stage in fundamentals of digital image processing. After acquired an image, various image processing methods are applied on images for further processing. Here medical heart image data set is taken for processing to find out the disease of coronary heart artery [21], [22].

\subsection{Image Preprocessing}

This section includes the following important steps are

A. Removal of Shadow Reduction/Blood noise reduction.

B. Cartesian to Polar (C2P) Coordinate conversion.

\section{A. Removal of shadow reduction/Blood noise reduction}

Shadow/blood noise areas usually do not represent any useful information in grey scale IVUS image. Therefore, this method has two types of thresholds namely High threshold and low threshold. Thresholding techniques are used to detect the low intensity regions following the high intensity regions, because 
the very high intensity regions are might belong to the plaque regions of IVUS image.

If this system denote intensity of the pixel I $(i, j)$ within the input image and the pixel $\mathrm{x}$ is the shadow region and it can be defined as follows:

$\mathrm{x}=(\mathrm{i}, \mathrm{j}) € \sum$

If intensity of pixel < low threshold

and $\mathrm{I}(\mathrm{i}, \mathrm{j} \pm 1) \varepsilon \sum$

If intensity of pixel < low threshold and $\mathrm{I}(\mathrm{i}, \mathrm{j} \pm 1)>$ high threshold
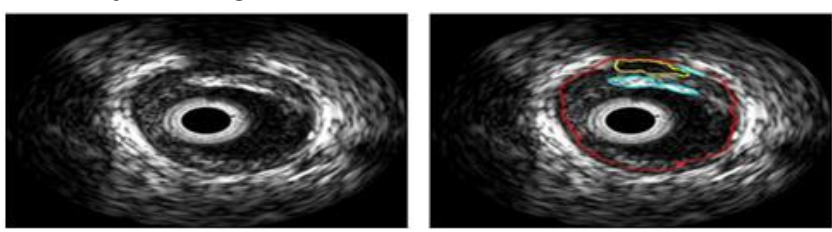

Figure 2: Separation of border detection from the background

Figure 2 illustrates two sample images from the dataset, here the yellow line indicates the shadow region and red line indicates the border detection. The threshold values are selected based on the mean value of the intensities in the shadow regions like high threshold and low threshold correspondingly. The resulting threshold values are initially normalized into statistical unity like zero mean and standard deviation for generalization purpose.

\section{B. Cartesian to Polar (C2P) Coordinate conversion}

Coordinate conversion is the important for describing the local image regions. So, the input image is convert from Cartesian to polar domain format which is shown in figure 3, where rows and columns are corresponding to the angle and distance from the center of the catheter device space which is denoted as $\mathrm{I}(\mathrm{r}, \theta)$ as shown in eq 3 .

Algorithm 1 Angle Decomposition of IVUS Image regions

Step (1) Input : IVUS image size of 512 x 512 pixels

Step (2) img : bidimensional image.

Step (3) Radius : radius length (\# of pixels to be considered).

Step (4) Angle : \# of angles to be considered for decomposition.

Output: pcimg: polar coordinate image.

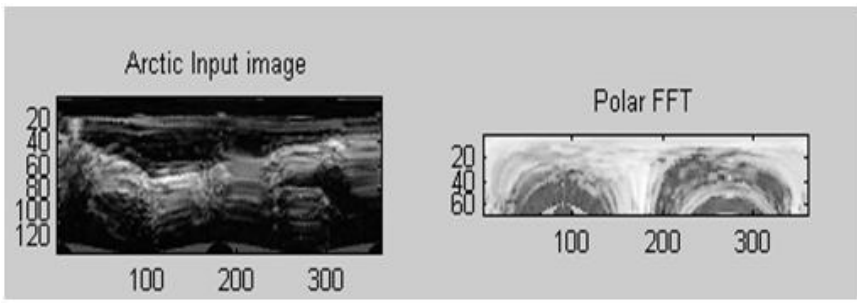

Figure 3: Transformation of IVUS image regions

limage (rradial, $\theta$ tangential)

It is also facilitates the number of detection steps for Ultrasound medical images such as contour initialization and refining of the obtained contour in IVUS image Regions and the algorithm 1 is used to describe the angle decomposition for coordinate conversion.

\subsection{Iterative level Energy Computation Method (IECM)}

IECM consists of Region-based geometric contour models are used to detect the connected pixels that employ the region information from the IVUS images. All possible integer threshold values are binarize and analyze the local edge base results are obtained by IECM. IECM contains the two important steps of region based geometric contours which are extracted from the input image.

Algorithm 2 explains the first step includes the region that evolves from brighter surfaces to darker boundaries is identified by the variable C1-. The superscript '-'symbol indicates that the brightness point of an image values. The values decreased from the surface of the image regions towards the boundaries of an IVUS image. The second step consists of region that evolves from darker surfaces with brighter boundaries and is denoted by $\mathrm{C} 2+$. The above said region is used to visualize the characteristics of IVUS image layers (Lumen and media). Therefore, IECM need to extract the $\mathrm{C} 2+$ regions are used to obtain the information about the lumen and media because both the regions evolve from dark surfaces with brighter boundaries.

Algorithm 2 Working Procedure of IECM

Input image with initial contour

Computes $\mathrm{C} 1, \mathrm{C} 2$, average prototypes of regions

for inside and outside of the contour

New degree of membership value is calculated

Difference $\Delta \mathrm{f}$ between new and old energy is calculated

Calculate total energy $\mathrm{F}$

Output: Get a segmented image 
S. Sridevi et al., International Journal of Advanced Trends in Computer Science and Engineering, 9(3), May - June 2020, 3877 - 3886

\subsection{Feature Extraction}

In feature extraction stage, most important key points are captured and extracted from IVUS images. In any segmentation approach considerable number of normal objects is recognized as pathological, which results in reduced efficiency of the detection system. However, one of the most important problems of IVUS imaging system is selection of standard feature set and the classification method, which is used to extract Region of Interest (ROI) while minimizing the false positive findings. Feature extraction technique and its corresponding features are reported in Table 1.

Table 1: Feature extraction techniques with corresponding feature

\begin{tabular}{|l|}
\hline \multicolumn{1}{|c|}{ Feature extraction techniques } \\
\hline Shape: X-coordinate maximum length of the plaque image, \\
y-coordinate maximum length of the plaque image, area of \\
ROI, Perimeter of ROI. Eccentricity of Lumen. \\
\hline SIFT: Finding Harris corners, Calculating characteristic \\
scale Calculating main orientation, Calculating descriptors, \\
Plotting/rotating matches. \\
\hline LBP: Relation between pixels and its neighbors. \\
\hline
\end{tabular}

\subsubsection{Shape Features}

Shape features are mainly used to extract the following features to identifying the exact plaque regions in a coronary artery. The features are like $\mathrm{x}$ and $\mathrm{y}$ coordinates maximum length, Region of Interest (ROI), perimeter of ROI and Eccentricity value calculation.

The above said features are providing essential information to classify the heart disease dataset according to the mathematical calculation shown in following equations.

$$
\begin{aligned}
& \mathrm{D}=2 \sqrt{C 5 A} / \pi \\
& \mathrm{CSF}=(\pi \cdot \mathrm{D} / \text { observed perimeter })^{2} \\
& \mathrm{~S}_{\mathrm{CSA}}=\left(\left(\mathrm{CSA}_{\mathrm{m}}-\mathrm{CSA}_{\mathrm{I}}\right) / \mathrm{CSA}_{\mathrm{m}}\right) \times 100 \\
& \text { (6) }
\end{aligned}
$$$$
\text { LLMAXD-LLMIND/100 }
$$

\subsubsection{SIFT Features}

In this approach introduces the Nearest Neighbor approach with high probability Euclidean metric based algorithm for matching the feature points in an image. In Cartesian coordinates, if $\mathrm{p}=$ $(\mathrm{p} 1, \mathrm{p} 2, \ldots \mathrm{pn})$ and $\mathrm{q}=(\mathrm{q} 1, \mathrm{q} 2, \ldots \mathrm{qn})$ are two points in
Euclidean two dimensional space, then the distance (d) vector from $\mathrm{p}$ to $\mathrm{q}$, or form $\mathrm{q}$ to $\mathrm{p}$ is defined as

$$
\mathrm{d}(\mathrm{p}, \mathrm{q})=\mathrm{d}(\mathrm{q}, \mathrm{p})=\text { rootof }\left(\mathrm{q}_{1}-\mathrm{p}_{1}\right) 2+\left(\mathrm{q}_{2}-\mathrm{p}_{2}\right)^{2}+\ldots+\left(\mathrm{q}_{\mathrm{n}}-\mathrm{p}_{\mathrm{n}}\right)^{2}(8)
$$

\subsubsection{Nearest neighbor approach}

SIFT uses the nearest neighbor approach and it is mainly used for classification method, which should be the first choice for a feature extraction study, where there is little or no more prior knowledge about the distribution of pixels in an image shown in the following Figure $4-6$ and algorithm 3 .

\begin{tabular}{l}
\hline Algorithm 3 nearest neighbor approach \\
\hline NN (Image, Candidate points) \{ \\
Step (1) Go through each candidate points in an image, \\
Step (2) Calculate the "distance" from that image to \\
specific sample candidate points. \\
Step (3) Out of those candidate points values pick the "k" \\
points, $\quad$ maybe in a premade array of "k" $\}.$ \\
\hline Output: Get a Premade
\end{tabular}

In polar coordinates of the point $\mathrm{p}$ are (radial 1 characteristic (r1), tangential 1 charactrestic $(\theta 1)$ ) and those of q are (radial 2 characteristic ( $\mathrm{r} 2)$, tangential 2 characteristic $(\theta 2)$ ) than the distance between the points are defined as follows.

$$
\text { Polar point }=(\mathrm{R} 2+\mathrm{r} 2-2 \mathrm{r} 1 \mathrm{r} 2 \operatorname{COS}(\theta 1-\theta 2))^{2}
$$

Figure 7 explains the Image feature points and also detected by using the Gaussian difference function, thus providing the similarity invariance between corresponding points of an image and also creating the feature descriptor by using the common geometric transformation.

Scale invariant interest point detector consists of four parameters like initial Gaussian blur, steps per scale octave, minimum image size and maximum image size. Local image interest points are matched by using local image descriptor. It contains the following similar descriptors are feature descriptor orientation bins, feature descriptor size, closest or next closest ratio. Local image feature descriptions are filtered by using the geometric filter, which are maximum alignment error, minimal inliers ratio, and expected transformation. 
S. Sridevi et al., International Journal of Advanced Trends in Computer Science and Engineering, 9(3), May - June 2020, 3877 - 3886

\subsubsection{LBP Features}

Local Binary Pattern (LBP) is a type of feature used in classification. Local feature encode for texture information of IVUS images, Table 2 explains the feature vector information which can use for IVUS image classification to obtaining the region information. The function portioning input image information for non-overlapping cells. The working procedure of LBP method is shown in algorithm 4.

\begin{tabular}{l}
\hline Algorithm 4 Generation of LBP Feature Vector \\
\hline Step (1) The number of cell is calculated by using the \\
Numcells = prod (floor (size (I) cellsize)) \\
Step (2) Feature length calculation using \\
LBP feature N=Numcells x B \\
LBP Features = size $\left(\mathrm{L}_{\mathrm{yx}}\right) \quad[1, \mathrm{~B}]$ \\
\hline Output: Get a Texture information \\
\hline
\end{tabular}

Table 2: LBP features for obtaining textual information about IVUS images

\begin{tabular}{|c|c|c|c|}
\hline $\begin{array}{c}\text { Input } \\
\text { Arguments }\end{array}$ & $\begin{array}{c}\text { Algorithm } \\
\text { Parameter }\end{array}$ & $\begin{array}{c}\text { Histogram } \\
\text { Parameter }\end{array}$ & $\begin{array}{c}\text { Output } \\
\text { Arguments }\end{array}$ \\
\hline \multirow{5}{*}{ Input } & Number of & & Features \\
& neighbors & Normalizatio & \\
\cline { 2 - 2 } image & select & $\mathrm{n}$ & Call for \\
& neighbors & Interpolation & number \\
& & used to & of cells \\
\cline { 2 - 2 } & Upright & compute & Call for \\
& Rotation & pixels. & LBP \\
& Invariance & & Feature \\
& Flag) & & Length \\
& & & \\
\hline
\end{tabular}

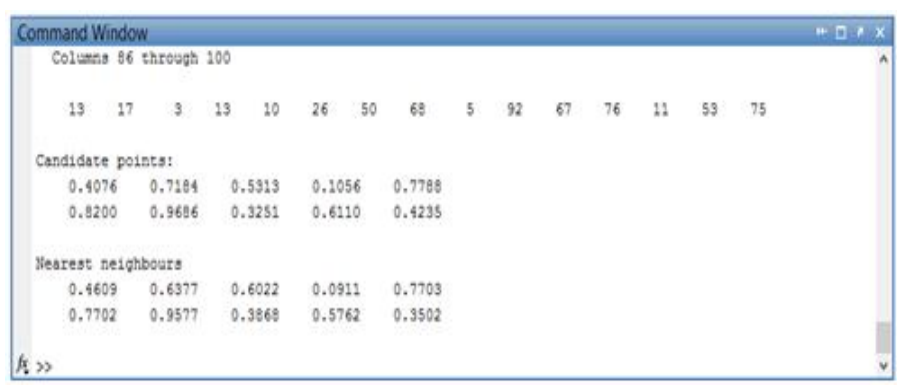

Figure 4: Selection of Nearest Neighbor

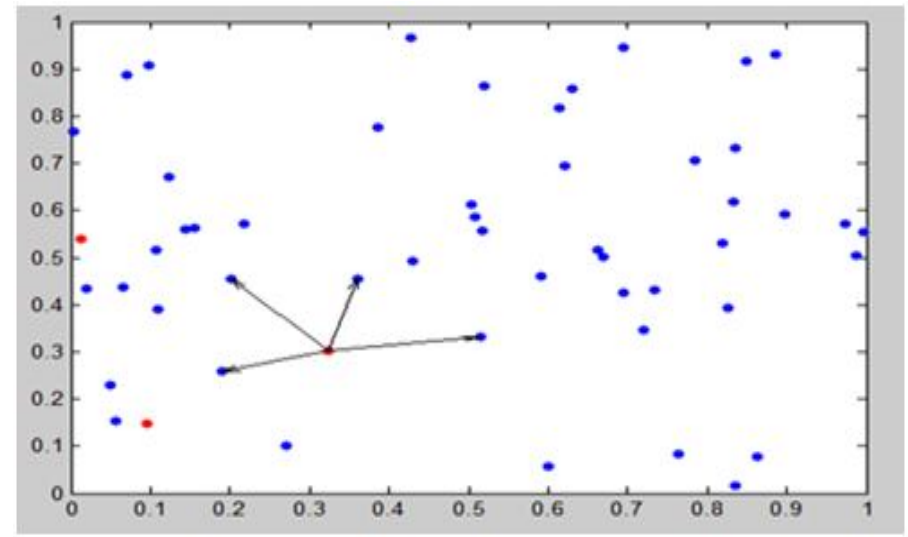

Figure 5: Finding nearest neighbor approach with specified radius

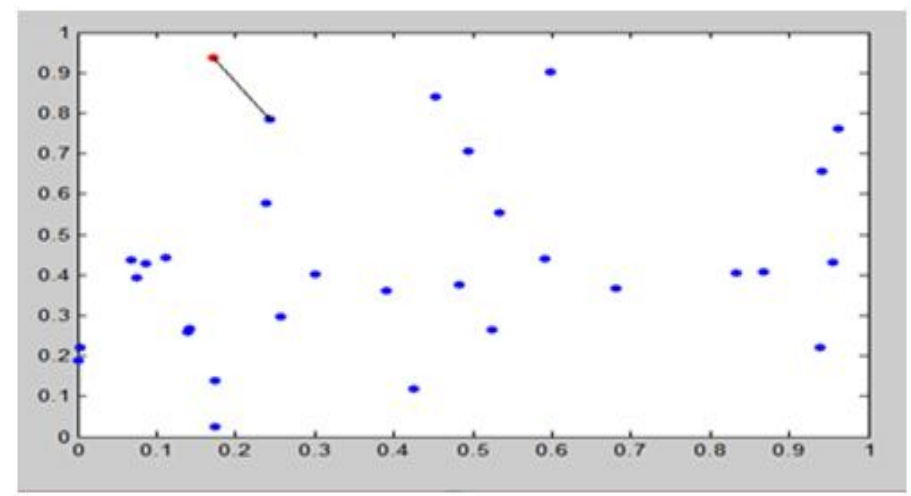

Figure 6: Combining number of neighbors and radius

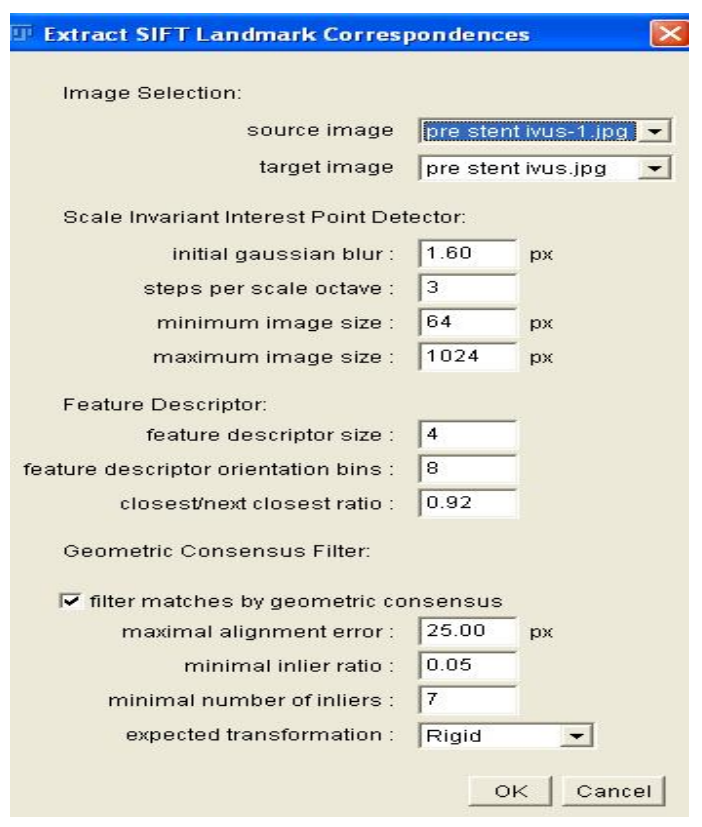

Figure 7: Enhancement of SIFT descriptor

\subsection{Classification}

In classification SVM uses the Modified Radial Basis Function Kernel (MRBFK). To avoid the misclassification 
S. Sridevi et al., International Journal of Advanced Trends in Computer Science and Engineering, 9(3), May - June 2020, 3877 - 3886

error, the training classifier finds out the optimal hyper plane and it is used to map the input vector elements into higher dimensional space where a hyper plane can separate the data into different classes (Actual and Predicted classes).

The Radial basis function is mainly handling the non-linear relations with classes and their feature vectors and then normalization was applied into feature vectors $[0,1]$ before applying the classifier. Standard deviation and the radial basis kernel were selected based on the feature selection. The kernel function considering two parameters namely $\mathrm{k}\left(\mathrm{x}_{\mathrm{i}}, \mathrm{x}\right)$ and $\gamma_{\mathrm{i}}$. The $\mathrm{k}$ is the kernel parameter and $\gamma_{\mathrm{i}}$ is the error term. Testified results were obtained in order to set up the kernel parameter is shown in Figure 8.

MRBFK represents the value of inner product of two training points in feature space. Kernel function uses the two steps which is mapping and do inner product in the feature space. The Modified Radial Basis Function Kernel represented as feature vector in some input space and used to maps the samples namely $\alpha_{\mathrm{i}}$ and $\mathrm{x}_{\mathrm{i}}$, is defined as

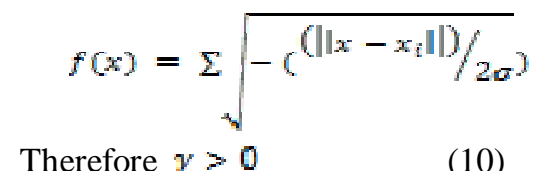

\subsubsection{SVM Classifier with MRBFK}

Modified Radial Basis Function Kernel is used to performing the models are easier to finding the best parameters place on the IVUS image boundaries of the grid, and also kernel parameter can be extended up to subsequent search. Algorithm 5 consists of two steps, Step 1 initially, is used to simplified classification problem involving the input features (Shape, SIFT and LBP) and 2 possible target classes (actual and predicted class). Finally, decision functions are visualized for variety of parameter values. Step 2 consists of mapping the classifiers of cross validation accuracy as a function $\mathrm{C}$ and gamma. This paper found the effectiveness of the SVM equipped with MRBFK to improve the precision and studied the specificity with respect to the parameter $\mathrm{k}$ which controls the degree of the conformal transform is defined as follows

$$
\begin{aligned}
& f(x)=\sum i^{\wedge} \operatorname{N\alpha i\gamma ik}(x i, f(x)+\beta \\
& \left.f(x)=\sum i^{\wedge} N \alpha i \gamma i \exp \sqrt{ }\left(-\left(\left(||\left(\left|x-x \_i\right|\right) \mid\right)\right) 2 \sigma\right)\right)+\beta
\end{aligned}
$$

Algorithm 5 SVM - MRBFK Parameters cross validation procedure

Classification begins......

(1) ClassMidpointNormalize(Normalize);

(2) load and prepare the dataset

(3) Dataset for grid search

(4) $x=$ IVUS.data

(5) $\mathrm{y}=$ IVUS.target

(6) Dataset for decision function visualization: features in $\mathrm{x}$ and subsamples to keep only 2 classes.

(7) $\mathrm{x} \_2 \mathrm{~d}=\mathrm{X}[:,: 2]$

(8) Scale the data for SVM training

(9) Training set obtains the transformation from one coordinate to another. $\mathrm{x} \_2 \mathrm{~d}=$ scalar.fit.transformation(x_2d) \%for an initial search set the gamma range.

(10) Param _grid $=\operatorname{dict}($ gamma $=$ gamm_range, $\mathrm{C}=$ C_range)

(11) validate accuracy of proposed method MRBFK with the variable (gamma and C)

Proposed model of the SVM with Modified Radial Basis

Function Kernel

Step 0: Set the control parameters $C, \sigma, \mathrm{k}$

Step 1: Train SVM with a primary MRBFK defined by Eq 7

Get $\alpha \mathrm{i}, \mathrm{b}$ and the set of support vectors and determine the decision boundary by $\mathrm{f}(\mathrm{x})=\sum \gamma \mathrm{i \alpha iK}(\mathrm{x}, \mathrm{xi})+\mathrm{b}$ iesv

Step 2: given $\mathrm{f}(\mathrm{x})$, train the modified RBFK SVM by Eq 8 For the same data and get the new decision boundary $\dot{\mathrm{f}}(\mathrm{x})$

Step 3: based on $\dot{\mathrm{f}}(\mathrm{x})$ and newly observed state is obtained.

Output: Calculate the prediction and store the result.

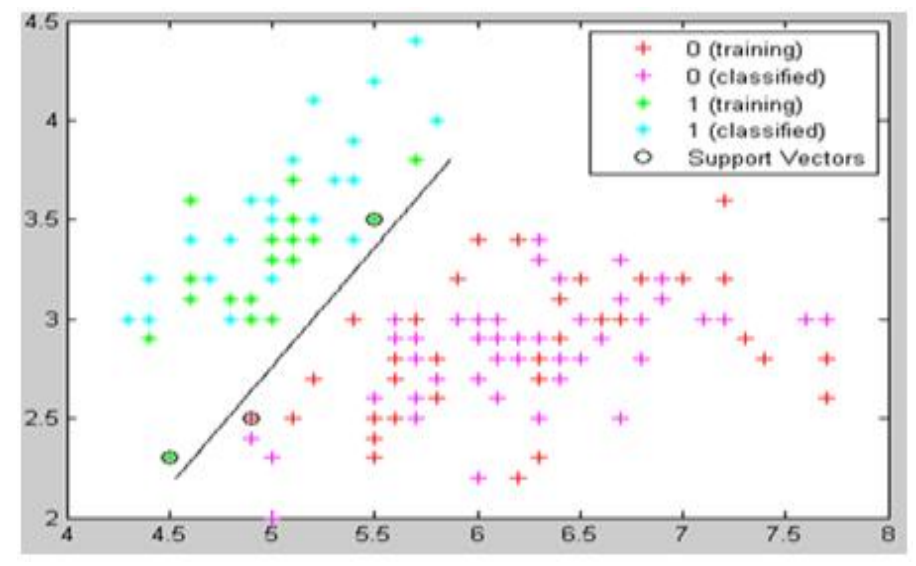

Figure 8: MRBFK Creation using feature vectors based on training and testing set. MRBFK, Modified Radial Basis Function Kernel 
S. Sridevi et al., International Journal of Advanced Trends in Computer Science and Engineering, 9(3), May - June 2020, 3877 - 3886

\subsubsection{Statistical Analysis}

In this section the specificity, sensitivity and accuracy of classifier validation studies are calculated. In addition the Kappa Statistic, Area under the Curve (AUC) and Confidence Interval (CI) level are also computed from the above said parameters for quantifying the degree of agreement defined as follows.

Kappa Value $=(($ observed - expected $)$ Proportion $/ 1-$ expected proportion).

Area Under the Curve $y=f(x)$ between $x=a \& x=b$, Integrate $\mathrm{y}=\mathrm{f}(\mathrm{x})$ between the limits $\mathrm{a}$ and $\mathrm{b}$.

\subsubsection{Performance Evaluation}

In the proposed method used to evaluate the performance through statistical analysis. For this, True Positive (TP), False Positive (FP), False Negative (FN) and True Negative (TN) and then Sensitivity, Specificity, Precision and Accuracy are calculated and shown in Table 3.

$$
\mathrm{CI}(0.86 \%)=\mathrm{X} \pm 1.96 \times \mathrm{Xx}(1-\mathrm{X}) / \mathrm{N}
$$

\section{RESULTS AND DISCUSSION}

In this section, the performance of the proposed method is presented. First, the performance of shadow detection procedure investigated and corresponding segmented IVUS images are analyzed by the proposed method. A sensitivity of 93.6 and specificity of 96.6 is obtained by comparing manually and automatically detected regions. After shadow detection the Shape, Scale Invariant Feature Transform features and Local Binary Pattern features were detected from the remaining portion of the plaque area.
Table 3: Pseudo code for performance metrics (TP, FN, TN, and TP)

\begin{tabular}{|c|c|c|c|}
\hline $\begin{array}{c}\text { True } \\
\text { Positive }\end{array}$ & $\begin{array}{c}\text { False } \\
\text { Negative }\end{array}$ & $\begin{array}{c}\text { True } \\
\text { Negative }\end{array}$ & $\begin{array}{c}\text { False } \\
\text { Positive }\end{array}$ \\
\hline $\begin{array}{l}\text { Procedure: } \\
\text { Calculate } \\
\text { the True } \\
\text { Positive as } \\
\text { follows. } \\
\text { True } \\
\text { Positive } \\
\text { Rate= TP / } \\
\text { (TP + FP). } \\
\text { I - Read } \\
\text { image, T - } \\
\text { Ground } \\
\text { truth } \\
\text { image. } \\
\text { TP=1 } \\
\text { For } \mathrm{i}: \mathrm{m} \\
\text { step of } 1 \\
\text { For } \mathrm{j}: \mathrm{n} \\
\text { step of } 1 \\
\text { If } \mathrm{I}(\mathrm{i}, \mathrm{j})=1 \\
\text { and } \mathrm{T}(\mathrm{I}, \mathrm{j}) \\
=1 \text { then } \\
\text { TP = TP } \\
+1, \text { print } \\
\text { True } \\
\text { Positive } \\
(\mathrm{TP})\end{array}$ & $\begin{array}{l}\text { Procedure: } \\
\text { calculate } \\
\text { the False } \\
\text { Negative } \\
\text { as follows: } \\
\text { Flase } \\
\text { Negative } \\
\text { Rate = } \\
\text { FN / (FN + } \\
\text { TP). } \\
\text { I - Read } \\
\text { image, } \mathrm{T}- \\
\text { Ground } \\
\text { truth } \\
\text { image. } \\
\text { FN = } 0 \\
\text { For i:m } \\
\text { step of } 1 \\
\text { For j: } \mathrm{n} \\
\text { step of } 1 \\
\text { If (i, } \mathrm{j})=1 \\
\text { and T(i, } \mathrm{j} \text { ) } \\
=0 \text { then } \\
\text { FN = FN + } \\
1, \text { print } \\
\text { False } \\
\text { Negative } \\
\text { (FN). }\end{array}$ & $\begin{array}{l}\text { Procedure: } \\
\text { Calculate } \\
\text { the True } \\
\text { Negative } \\
\text { as follows: } \\
\text { True } \\
\text { Negative } \\
\text { Rate= TN / } \\
\text { (TN + } \\
\text { FN). } \\
\text { I - Read } \\
\text { image, T - } \\
\text { Ground } \\
\text { truth } \\
\text { image. } \\
\text { TN=1 } \\
\text { For i:m } \\
\text { step of } 1 \\
\text { For j:n step } \\
\text { of } 1 \\
\text { If I(i, j) =0 } \\
\text { and T(i, j) } \\
=0 \text { then } \\
\text { TN = TN + } \\
1, \text { print } \\
\text { True } \\
\text { Negative } \\
\text { (TN). }\end{array}$ & $\begin{array}{l}\text { Procedure: } \\
\text { Calculate } \\
\text { the False } \\
\text { Positive as } \\
\text { follows: } \\
\text { Flase } \\
\text { Positive } \\
\text { Rate = FP / } \\
\text { (FP + TN) } \\
\text { I - Read } \\
\text { image, T- } \\
\text { Ground } \\
\text { truth } \\
\text { image. } \\
\text { FP = 0 } \\
\text { For i:m } \\
\text { step of } 1 \\
\text { For j: } n \\
\text { step of } 1 \\
\text { If (i, j) }=1 \\
\text { and T(i, j) } \\
=0 \text { then } \\
\text { FP = FP + } \\
1, \text { print } \\
\text { Flase } \\
\text { Positive } \\
\text { (FP). }\end{array}$ \\
\hline $\begin{array}{l}\text { Condition: } \\
\text { TP Rate= } 1 \\
\text { when FP = } \\
0 . \\
\text { (No false } \\
\text { positives) }\end{array}$ & $\begin{array}{l}\text { Condition: } \\
\text { FN Rate = } \\
0 \text { when } \\
\text { FN }=0 . \\
\text { (No false } \\
\text { negatives) }\end{array}$ & $\begin{array}{l}\text { Condition: } \\
\text { TN Rate = } \\
\text { 1when } \\
\text { FN }=0 . \\
\text { (No false } \\
\text { negatives) }\end{array}$ & $\begin{array}{l}\text { Condition: } \\
\text { FP Rate = } \\
0 \text { when FP } \\
=0 . \\
\text { (No false } \\
\text { positives) }\end{array}$ \\
\hline
\end{tabular}

Sensitivity, Specificity, Precision and Accuracy parameters are calculated in order to assess the performance of the proposed method. Figure 9 shows the calculation of three statistical parameters which are sensitivity, specificity and precision. Figure 10 shows accuracy is calculated based on the Parameters like true positive, false positive, false negative and true negative.

Figure 11 shows the Confusion matrix of MRBF Kernel creation. Confusion matrix is a special kind of contingency table which allows the visualization of the performance of a 
method. It is a specific table layout, with two dimensions ("actual" and "Predicted"), and identical sets of "classes" in both dimensions. Confusion matrix shows the performance of the proposed Modified Radial Basis Function Kernel (MRBFK) which are effectively used in higher dimensional space to detect the misclassification errors in training and testing samples.

The Classification accuracy is reported in table 4. Classifier that gives nearly similar accuracies which shows the reliability of the proposed image based validation method.

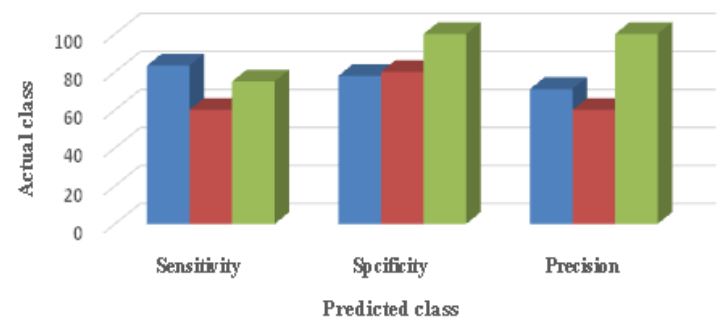

Figure 9: Graphical representation chart for evaluation of performance of classification parameter like Sensitivity, Specificity and Precision

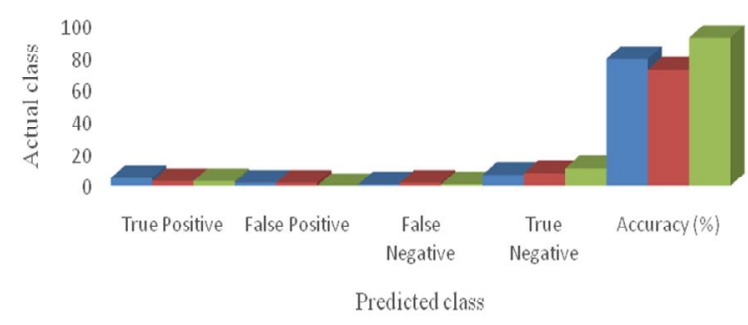

Figure 10: Graphical representation chart for evaluation of performance of classification parameter like True positive, False positive, True Negative and False Negative

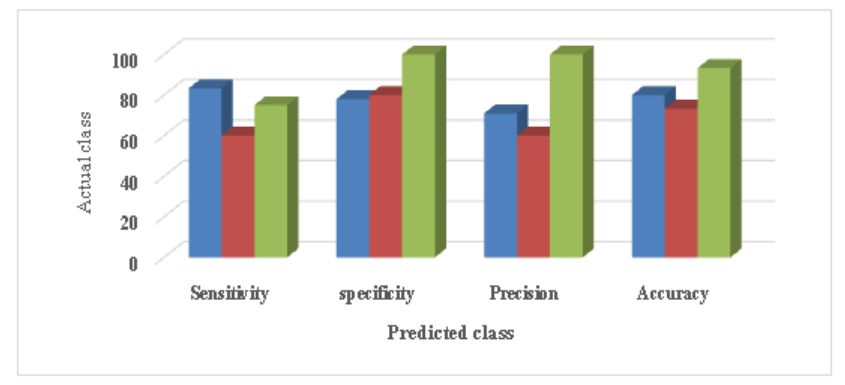

Figure 11: Graphical representation chart for confusion matrix of SVM classifier with MRBFK based on the metrics like True Positive, False Positive, False Negative, True Negative and Accuracy

\subsection{Comparison Results}

The Existing and proposed method and its corresponding metric based analysis are reported in the table 4 . According to the feature extraction, results based classification results are validated and also the proposed method performance are reported and compared with the existing methods.

Existing Method 1 (SVM) and its corresponding metrics (Sensitivity, Specificity, Precision and Accuracy) is evaluated. Overall accuracy is $74 \%$.

Existing Method 2 (ECOC) and its corresponding metrics (Sensitivity, Specificity, Precision and Accuracy) is evaluated. Overall accuracy is $84 \%$.

Proposed MRBFK and its corresponding metrics (Sensitivity, Specificity, Precision and Accuracy) are evaluated. Overall accuracy is $88 \%$. All the above results have been tabulated in Table 4 and have been shown graphically in Figure 12.

Table 4: Comparison results

\begin{tabular}{|c|c|c|c|c|}
\hline Classifier & $\begin{array}{c}\text { Sensitivity } \\
(\%)\end{array}$ & $\begin{array}{c}\text { Specificity } \\
(\mathbf{\%})\end{array}$ & $\begin{array}{c}\text { Precision } \\
(\%)\end{array}$ & $\begin{array}{c}\text { Accuracy } \\
(\%)\end{array}$ \\
\hline SVM & 75.0 & 99.1 & 60 & 74 \\
\hline ECOC & 85.3 & 94.8 & 71 & 84 \\
\hline $\begin{array}{c}\text { Proposed } \\
\text { SVM with } \\
\text { MRBFK }\end{array}$ & 93.6 & 96.6 & 81 & 88 \\
\hline
\end{tabular}

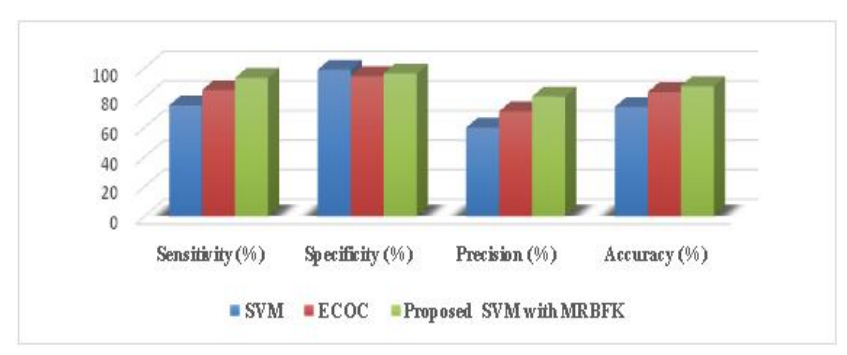

Figure 12: Graph representation of comparison result

\section{CONCLUSION AND FUTURE ENHANCEMENT}

In this paper, an effective method for the challenging problem of Coronary Heart Artery Disease classification was presented. Several combining features were produced in this work.

The SVM with MRBFK is used to describe the reliability and projection into this infinite higher dimensional space where the data becomes linearly separable. Then, the Radial 
Basis Function Kernel is used when the boundaries are hypothesized to be curve-shaped. Another contribution of this paper investigates thoroughly on new data sets and also asses the reliability of the reported results.

The results supporting the positive effects of combining the three feature extraction methods. And then, generates the multi - resolution characteristics of more detailed images performs better. Furthermore, the results obtained might be the $(88 \%)$ highest achievable accuracy, using the combination of image based feature extraction method.

In future research, there is a need and huge scope to improve the Dice coefficient, Recall, and F-measure in order to perform the classification of IVUS images.

\section{REFERENCES}

1. Arash taki,Holger hetterich and et al. A New approach for Improving Coronary Plaque Component Analysis Based on Intravascular Ultrasound Images, Elsevier, Ultrasound in Med. \& Biol., Vol. 36, No. 8, pp. 1245-1258, 2010.

2. Archana K.V.,.Vanithamani R. Review on Intravascular Ultrasound Image Detection, International Journal of Advanced Research in Science, Engineering and Technology, Vol.4, Issue 7, 2017.

3. Ashok kumar L., and P. Rajendran. Intima-media thickness segmentation using weighted graph based active contour technique, Biomedical Research, 1-1, 2017"

4. Christodoulou C I, Pattichis C.S. Image Retrieval and Classification of Carotid Plaque Ultrasound Images The Open Cardiovascular Imaging Journal, 2, 18-28, 1876-5386, 2010. https://doi.org/10.2174/1876538601002010018

5. Daisuke Nagatomo, Daizaburo Yanagi, and Takeshi Serikawa. The Role of Intravascular Ultarsound scan and thin-sliced coronary computed tomography angiography in diagnosing aortic dissection causing acute myocardial infarction, International Journal of Case Reports and Images, vol.5 no 4, 2014.

6. Escalera S, Pujol O, and PetiaRadeva. ECOCs library, J. Mach. Learn. Res. 11 661-664, 2010.

7. Ga Young kim, Ju Huwan Lee. A Novel Intensity based Multi level Classification approach for Coranry Plaque Characterization in Intravascular Ultrasound Images, Biomed Engineering online, DOI:10.1786/S12938-018-0586-1, 2018.
8. Hassen Lazrag, Kamel Aloui, and Med Saber Naceur. Automatic Segmentation of Lumen in Intravascular Ultrasound images using Fuzzy Clustering and Active Contours, International conference on control, engineering \& Information Technology (CEIT '13) Proceedings Engineering \& Technology- Vol.1, 2013.

9. Luo Z, Wang Y, and Wang W, Estimating coronary artery lumen areawith optimization-based contour detection, IEEE Trans Med Imaging, 22(April):546-64, 2013.

https://doi.org/10.1109/TMI.2003.811048

10. Sridevi S, Sundaresan M. Hybrid Feature Extraction Techniques For Accuracy Improvement In IVUS Image Classification, International Journal of Scientific \& Technology Research, Volume 9, Issue 04, ISSN-2277-8616, 2020.

11. Sun $\mathrm{Z}$, Liu $\mathrm{C}, A$. parallel method for segmenting intravascular ultrasound image sequence. Appl Mech Mater, 130:2051-5, 2011.

12. Syaiful Anam, Eiji Uchino and Noriaki suetake. Hybrid Boundary Detection Method for Image with Application to Coronary Plaque, International Journal of Digital Information and Wireless Communications (IJDIWC), Vol. 4, 2014.

13. Topol E.J. Textbook of Cardiovascular Medicine, Lippincott Williams \& Wilkins, 3rd edition, 2007

14. Xuan Liang, Michalis Xenos, Yared Alemu, Suraj H and et al. Biomechanical Factors in Coronary vulnerable Plaque Risk of Rupture: Intravascular Ultarsound-based Patient- Specific fluid - structure interaction studies, Pathophysiology and natural history, Vol 24, No2, 2013.

15. Selvathi D, Emimal N. Performance analysis of contourlet Features with SVM classifier for the characterization of Atheromatous plaque in Intravascular Ultrasound images, Journal of Engineering Research and Applications, ISSN: 2248-9622, vol.4, Issue 3(Version 4), pp.35-40, 2014.

16. David Molony, Habib Samady. TCT-342 Deep IVUS: A Machine Learning Platform for Fully Automatic IVUS Segmentation and Phenotyping, Journal of the American College of Cardiology, Volume 74, Issue 13 Supplement, DOI: 10.1016/j.jacc.2019.08.424, 2019.

17. Ji Yang, Mehdi Faraji. Robust Segmentation of Arterial Walls in Intravascular Ultrasound Images Using Dual Path U-Net, Ultrasonics National Library of Medicine, DOI: 10.1016/j.ultras.03.014, 2019.

18. Lucas Lo Vercio, Jose Ignacio Orlando, Mariana del Fresno, and Ignacio Larrabide. Assessment of image 
S. Sridevi et al., International Journal of Advanced Trends in Computer Science and Engineering, 9(3), May - June 2020, $3877-3886$

features for vessel wall segmentation in Intravascular Ultrasound images, Article in International Journal of Computer Assisted Radiology and Surgery 11(8), DOI: 10.1007/s11548-015-1345-4, 2016.

19. VaishaliNaik, R.S.Gamad and P.P Bansod. The Carotid Intima media Thickness Measurement of Ultrasound images", Proceedings of the 12th INDIACom, INDIACom-2018; IEEE Conference ID: 42835, ISBN 978-93-80544-28-1, 2018

20. Huang Yi; Yan, Wenjun; and Xia, Menghua. Segmentation of Media and Lumen in Intravascular Ultrasound Image using Guided Multiscale Normalized Cut, Journal of Medical Imaging and Health Informatics, Volume 9, Number 7, 361-364, 2019.

21. B. Jabber.at.al, "A Novel Sampling Approach for Balancing The Data and Providing Health Care Management System by Government", International Journal of Advanced Trends in Computer Science and Engineering, Vol 8, No. 6,November -December 2019, $2753-2761$.

https://doi.org/10.30534/ijatcse/2019/12862019

22. Mani V, GokulPrasath R, Jegatha S, et al, "Hospital Recommendation System using Machine Learning", International Journal of Advanced Trends in Computer Science and Engineering, Volume 9, No. 2, March-April 2020, https://doi.org/10.30534/ijatcse/2020/64922020. 